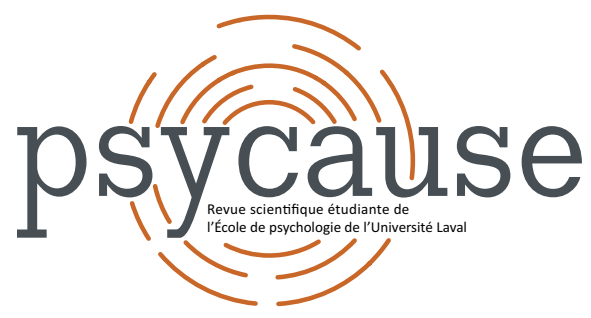

\title{
PSYCAUSE
}

Revue scientifique étudiante de l'École de psychologie de l'Université Laval

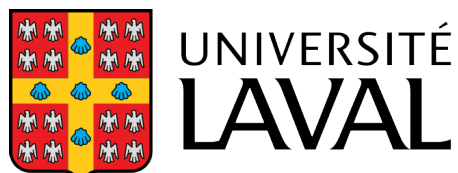

Faculté des sciences sociales École de psychologie

\section{AGRESSIONS SEXUELLES CHEZ L'ENFANT : SYMPTÔMES DE STRESS POST-TRAUMATIQUE ET COMPORTEMENTS SEXUELS PROBLÉMATIQUES}

Emma LAFLAMME ${ }^{1 *}$, Amée LAPOINTEE ${ }^{1}$, Claudia SAMSON ${ }^{1}$, Gabriel MARTIN-GAGNON ${ }^{1}$ et Karin ENSINK ${ }^{1}$

${ }^{1}$ École de psychologie, Université Laval

*emma.laflamme.1@ulaval.ca

\section{Pour citer l'article}

Laflamme, E., Lapointe, A., Samson, C., Martin-Gagnon, G., \& Ensink K. (2021). Agressions sexuelles chez l'enfant: symptômes de stress post-traumatique et comportements sexuels problématiques. Psycause: Revue scientifique étudiante de l'École de psychologie de I'Université Laval, 11(2), 5-6. 


\title{
AGRESSIONS SEXUELLES CHEZ L'ENFANT : SYMPTÔMES DE STRESS POST-TRAUMATIQUE ET COMPORTEMENTS SEXUELS PROBLÉMATIQUES
}

\author{
Emma LAFLAMME*, Amée LAPOINTE1 ${ }^{1}$, Claudia SAMSON¹, Gabriel MARTIN-GAGNON¹ et Karin ENSINK \\ ${ }^{1}$ École de psychologie, Université Laval \\ *emma.laflamme.1@ulaval.ca
}

\author{
Mots-clés: agressions sexuelles à l'enfance, trouble de stress post-traumatique, comportements sexuels \\ problématiques, enfants
}

Les enfants victimes d'agressions sexuelles sont davantage prédisposés au développement d'un trouble de stress post-traumatique (TSPT; Andrews et al., 2004; Easton et Kong, 2017). Selon Pratchett et Yehuda (2011), parmi tous les évènements traumatiques, I'agression sexuelle chez l'enfant est perçue comme l'un des plus importants facteurs de risque au développement de difficultés psychologiques futures. Cela s'explique par le fait que l'agression sexuelle survient tôt dans la vie de l'enfant lorsque son développement neurobiologique est malléable (Pratchett et Yehuda, 2011). L'agression sexuelle chez l'enfant peut également mener au développement de comportements sexuels problématiques (CSP; Collin-Vezina et al., 2013). L'Association for the Treatment of Sexual Abusers (2006) définit les CSP comme des gestes initiés sur des parties sexuelles du corps par des enfants de 12 ans et moins, considérés inappropriés en fonction de la trajectoire normale et qui sont potentiellement néfastes pour l'enfant ou pour autrui (Chaffin et al., 2008). Les comportements sexuels sont également jugés comme problématiques s'ils sont répétitifs, coercitifs ainsi que persistants, malgré une intervention parentale (Chaffin et al., 2008; Ryan, 2000). Cependant, peu d'études rapportent les facteurs influençant l'apparition de CSP (Elkovitch et al., 2009). Il est donc pertinent de vérifier quels sont les facteurs, tels que les symptômes du TSPT, qui mènent au développement de CSP chez l'enfant à la suite d'une agression sexuelle. L'objectif de la présente étude est de vérifier le rôle médiateur des symptômes du TSPT dans le développement de CSP chez les enfants âgés de 5 à 12 ans ayant vécu une agression sexuelle.

\section{Méthode}

Les données utilisées dans ce projet de recherche proviennent d'une étude de plus grande envergure s'intéressant à l'agression sexuelle chez l'enfant. Les enfants victimes d'agressions sexuelles sont référés par différents intervenants qui travaillent à la Direction de la protection de la jeunesse, dans les centres de santé et services sociaux ou dans les écoles de la région de Québec. Les enfants n'ayant pas vécu d'agression sexuelle, formant le groupe contrôle, sont recrutés par l'entremise de dépliants et d'affiches publicitaires déposés dans les écoles, les garde- ries et services de santé. L'échantillon se compose de 256 dyades mère-enfant, dont 173 dyades dans lesquelles les enfants ont une probabilité élevée d'avoir des antécédents d'agressions sexuelles et 83 dyades contrôles dans lesquelles les enfants ne sont pas des victimes d'agressions sexuelles. Les dyades incluent des enfants âgés de 5 à 12 ans. Dans le cadre de cette étude, le Child Behavior Checklist (CBCL; Achenbach, 1991) a tout d'abord été administré aux mères. Ce questionnaire permet de mesurer divers comportements problématiques chez l'enfant âgé de 4 à 18 ans (Achenbach, 1991). Ensuite, le Child Sexual Behavior Inventory (CSBI), un questionnaire rapporté par le principal donneur de soins d'un enfant soupçonné d'avoir été agressé sexuellement, a été administré aux mères. Le donneur de soins évalue la fréquence de comportements sexuels normaux ou problématiques chez l'enfant âgé de 2 à 12 ans au cours des six derniers mois (Friedrich et al., 1992).

\section{Résultats et discussion}

Les résultats de la présente étude indiquent que les enfants victimes d'agressions sexuelles présentant davantage de symptômes du TSPT développent plus de CSP. En effet, $33 \%$ de la variance entre l'agression sexuelle chez l'enfant et le développement de CSP est expliquée par les symptômes du TSPT. Ainsi, les résultats suggèrent que les symptômes du TSPT ont un rôle médiateur dans la relation entre l'agression sexuelle et le développement de CSP tel qu'illustré à la Figure 1.

Les présents résultats concordent avec les conclusions rapportées dans l'étude de Wamser-Nanney et Campbell (2020) postulant que les symptômes du TSPT influencent considérablement le développement de CSP chez les enfants victimes d'agressions sexuelles. Les résultats de cette étude indiquent que les symptômes du TSPT constituent une cible d'intervention pertinente pour prévenir le développement de CSP. En ciblant les symptômes de TSPT, cette étude permettra donc d'améliorer le dépistage et la prévention des problèmes développementaux à la suite d'une agression sexuelle. 


\section{Figure 1}

Modèle de médiation décrivant les relations significatives entre l'agression sexuelle, les symptômes du trouble de stress post-traumatique et les comportements sexuels problématiques.

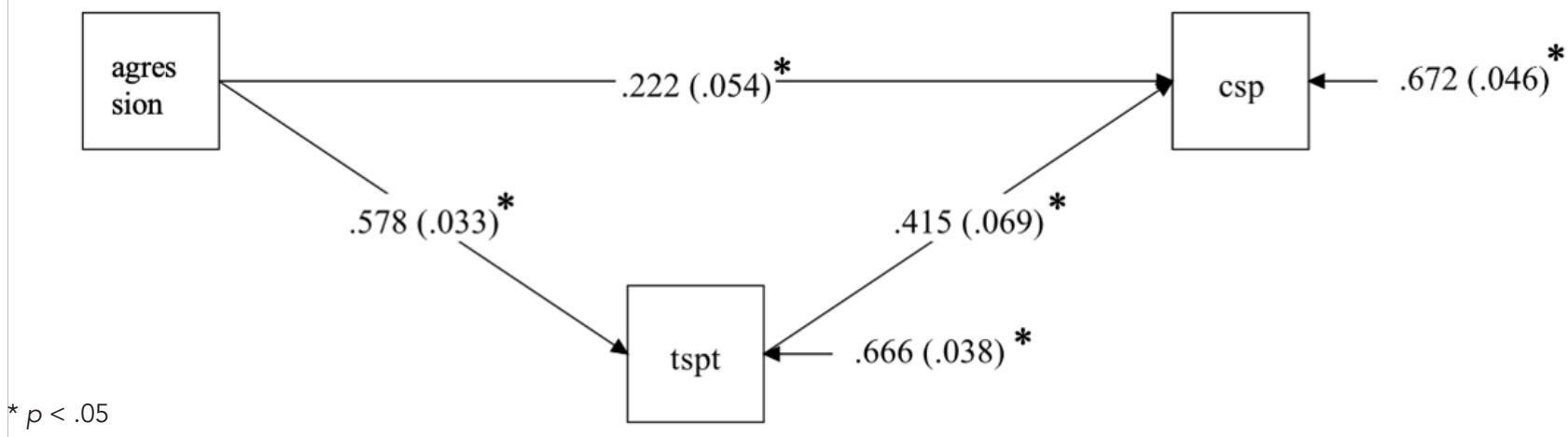

\section{Références}

Achenbach, T. M. (1991). Child Behavior Checklist. University of Vermont, Department of Psychiatry.

Andrews, G., Corry, J., Slade, T., Issakidis, C., \& Swanston, H. (2004). Comparative quantification of health risks: Global and regional burden of disease attributable to selected major risk factors. World Health Organization Geneva, 2, 1851-1940. http://citeseerx.ist.psu.edu/viewdoc/ download ?doi=10.1.1.948.7409\&rep=rep1\&type $=$ pdf\# page $=1875$

Association for the Treatment of Sexual Abusers. (2006). Report of the Task Force on Children with Sexual Behavior Problems. https://www.atsa.com/pdfs/Report-TFCSBP.pdf

Chaffin, M., Berliner, L., Block, R., Johnson, T. C., Friedrich, W. N., Louis, D. G., \& Madden, C. (2008). Report of the ATSA task force on children with sexual behavior problems. Child Maltreatment, 13(2), 199-218. https://doi. org/10.1177/1077559507306718

Collin-Vézina, D., Daigneault, I., \& Hébert, M. (2013). Lessons learned from child sexual abuse research: Prevalence, outcomes, and preventive strategies. Child and adolescent psychiatry and mental health, 7(1), 22. http://www.capmh. com/content/7/1/22
Easton, S. D., \& Kong, J. (2017). Mental health indicators fifty years later: A population-based study of men with histories of child sexual abuse. Child abuse \& neglect, 63, 273-283. https://doi.org/10.1016/j.chiabu.2016.09.011

Elkovitch, N., Latzman, R. D., Hansen, D. J., \& Flood, M. F. (2009). Understanding child sexual behavior problems: A developmental psychopathology framework. Clinical Psychology Review, 29(7), 586-598. https://doi. org/10.1016/j.cpr.2009.06.006

Friedrich, W. N., Grambsch, P., Damon, L., Hewitt, S. K., Koverola, C., Lang, R. A., Wolfe, V., \& Broughton, D. (1992). Child Sexual Behavior Inventory. https://doi.org/10.1037/ t00814-000

Pratchett, L. C., \& Yehuda, R. (2011). Foundations of posttraumatic stress disorder: Does early life trauma lead to adult posttraumatic stress disorder? Development and Psychopathology, 23(2), 477-491. https://doi.org/10.1017/ S0954579411000186

Ryan, G. (2000). Childhood sexuality: A decade of study. Part I-Research and curriculum development. Child abuse \& neglect, 24(1), 33-48. https://doi.org/10.1016/S01452134(99)00118-0

Wamser-Nanney, R., \& Campbell, C. L. (2020). Childhood sexual abuse characteristics, abuse stress, and PTSS: Ties to sexual behavior problems. Child abuse \& neglect, 105. https://doi.org/10.1016/j.chiabu.2019.104290

\section{Pour citer l'article}

Laflamme, E., Lapointe, A., Samson, C., Martin-Gagnon, G., \& Ensink K. (2021). Agressions sexuelles chez l'enfant: symptômes de stress post-traumatique et comportements sexuels problématiques. Psycause: Revue scientifique étudiante de l'École de psychologie de I'Université Laval, 11(2), 5-6. 